\title{
In-Band LOS Discovery Between Drones Using Highly Directional Transceivers
}

\author{
Mahmudur Khan \\ University of Central Florida \\ Orlando, Florida \\ mahmudur@knights.ucf.edu
}

\author{
Murat Yuksel \\ University of Central Florida \\ Orlando, Florida \\ Murat.Yuksel@ucf.edu
}

\begin{abstract}
Line-of-sight (LOS) establishment is a significant part of the neighbor discovery process for an ad-hoc network with highly directional antennas/transceivers. And, this problem of establishing LOS between neighbor nodes become even more challenging when they have no a priori information about each other's location. In this paper, we present a heuristic for LOS neighbor discovery between two drones capable of scanning the 3D space around them using highly directional transceivers. The drones can mechanically steer their respective transceivers with randomly chosen angular speeds on arbitrary circular paths and perform a simple three-way handshake to discover each other. They change the circular scanning paths and angular speeds if the discovery is not successful within an optimal time interval. Through extensive simulations, we demonstrate that using such mechanically steerable highly directional transceivers, two neighbor drones can discover each other successfully even without prior location information about each other and without any omnidirectional out-of-band channel to exchange information with each other.
\end{abstract}

\section{CCS CONCEPTS}

- Networks $\rightarrow$ Mobile networks; Ad hoc networks;

\section{KEYWORDS}

In-Band, Line-of-Sight, Neighbor Discovery, Drones, Directional, Free-Space-Optical, mmWave

\section{ACM Reference Format:}

Mahmudur Khan and Murat Yuksel. 2018. In-Band LOS Discovery Between Drones Using Highly Directional Transceivers. In DroNet'18: 4th ACM Workshop on Micro Aerial Vehicle Networks, Systems, and Applications , Fune 10-15, 2018, Munich, Germany. ACM, New York, NY, USA, 6 pages. https://doi.org/10.1145/3213526.3213536

\section{INTRODUCTION}

Highly directional transceivers are envisioned to play a major role in future generation wireless ad hoc networks [3]. These transceivers could be radio frequency (RF), mmWave or free-space-optical (FSO) transceivers. Directional transceivers provide larger transmission

Permission to make digital or hard copies of all or part of this work for personal or classroom use is granted without fee provided that copies are not made or distributed for profit or commercial advantage and that copies bear this notice and the full citation on the first page. Copyrights for components of this work owned by others than ACM must be honored. Abstracting with credit is permitted. To copy otherwise, or republish, to post on servers or to redistribute to lists, requires prior specific permission and/or a fee. Request permissions from permissions@acm.org.

DroNet'18, fune 10-15, 2018, Munich, Germany

(C) 2018 Association for Computing Machinery.

ACM ISBN 978-1-4503-5839-2/18/06.

https://doi.org/10.1145/3213526.3213536 range and enhance signal security by reducing the probability of jamming. They also reduce interference caused by unwanted sources. Moreover, directional transceivers improve spatial reuse and enable transfer of large volumes of data at very high speeds, e.g., 20Gbps [5]. All these features of directional transceivers are potentially very beneficial for future high-speed communications using unmanned aerial vehicles (UAVs) or quadcopters or drones [16].

UAVs or drones are also considered for both civil and military missions, such as monitoring of an area hit by a disaster, broadcasting data at some sports events or even observing behind the enemy lines. All these different applications of drones are imagined to produce large amounts of data to be delivered to a ground station or other drones [12]. Using directional transceivers, these large volumes of data can be transferred at extremely high speeds. Thus, communications among drones using highly directional transceivers is emerging as a promising technology. Google's Project Loon [6] aims at providing high-speed Internet to everyone, that includes the application of free-space-optical (FSO) communications among balloons. Another project aiming at connecting areas of the world lacking Internet infrastructure is 'internet.org' by Facebook. It includes a new technology using mmWave and laser based communication among solar powered drones (named Aquila) [5]. Due to high directionality, maintaining FSO links among drones using in-band techniques is challenging [10]. In-band discovery of such FSO links is even harder.

Communications using highly directional transceivers require strict maintenance of line-of-sight (LOS). Compared to the omnidirectional ones, these transceivers have very narrow field-of-view (FOV). Thus, for successful communication, two such transceivers must face directly towards each other. Hence, LOS discovery is the first important task for establishing a directional mmWave/FSO communication.

LOS (neighbor) discovery protocols can be classified into two broad categories:

(i) Synchronous and Out-of-Band: In [13], deployment of directional antennas in an ad hoc network is presented. The nodes are considered to be synchronized and could exchange location information acquired using GPS. In [18], an LOS discovery algorithm using directional transceivers is presented where all nodes use synchronized slots. The algorithms for LOS discovery presented in [7] and [9] consider using both directional transmitter and receiver, but requires synchronization among nodes.

(ii) Asynchronous and In-Band: An LOS discovery method with directional RF was proposed in [14] that requires no clock synchronization among nodes. It uses an optimal value of probability that depends on the node density of the network for transmitting beacon 
messages at random directions. In [2], [15] and [1], different LOS discovery protocols are presented for nodes in a $2 \mathrm{D}$ scenario. These protocols also assume no clock synchronization among the nodes. The nodes also have no a-priori information about each other's location. They only use directional antennas for neighbor discovery and work in a fully decentralized manner.

In this paper, we propose a novel method for LOS discovery between two nodes (e.g., hovering UAVs/drones/quadcopters) in a 3D environment We consider each node to be equipped with a highly directional FSO/RF transceiver, mounted on a mechanically steerable spherical structure/head. Thus, the transceiver can be steered to scan $360^{\circ}$ along the horizontal plane and $360^{\circ}$ along the vertical plane. We also assume that there is no GPS available to acquire location information. Moreover, we assume that there is no additional omnidirectional RF channel available to share location or any other information. The only way for the nodes to discover each other is to use the mechanically steerable highly directional transceivers to scan the surrounding 3D space in search of the neighbor node. Thus, in-band operation is the only viable option we consider while comparing to the cases with more information exchange capability between the nodes.

The gist of the neighbor discovery method is to scan the surrounding space by rotating the transceivers in randomly chosen circular paths. Each node arbitrarily chooses an angular speed and a circular path to start the discovery process. They start rotating their transceivers, and at the same time, start sending 'Hello' messages. When two nodes are within each other's coverage beam, they perform a three-way handshake and complete LOS discovery.

\section{TECHNICAL APPROACH}

\subsection{Assumptions}

We make the assumptions listed below for our proposed neighbor discovery method:

i) 3D environment: The nodes are situated in a 3D space, e.g., drones or UAVs hovering within each other's communication range.

ii) Directionality: The transceivers are highly directional. The nodes can communicate only while facing the transceivers towards each other.

iii) Scanning: The transceiver on each node is mounted on a mechanically steerable spherical structure with which it can scan $360^{\circ}$ in the horizontal plane $360^{\circ}$ along the vertical plane.

iv) In-band full-duplex: There is no additional omni-directional RF channel or GPS available. Only the highly directional transceivers can be used for communication. The nodes simultaneously transmit and receive over the same channel ${ }^{1}$.

v) Asynchronous algorithm: The nodes run the proposed discovery algorithm in a distributed manner without any synchronization mechanism.

vi) Transceiver beam: The transmitter and the receiver are placed closely together in the transceiver. So, we assume one beam pattern to indicate both the transmission's divergence angle and the reception's FOV. We approximate the beam with a cone

\footnotetext{
${ }^{1}$ Attaining this full-duplex operation can easily be done via isolation of transmitter and receiver [8]. There have also been several recent works showing the feasibility of full-duplex radio transceivers as well [11].
}

of height $r$ and radius $r \tan \beta$, where $r$ is the maximum communication range of the transceiver and $\beta$ is the divergence angle.

\subsection{Discovery}

Let us assume two nodes A and B are hovering within each other's communication range, and are unaware of each other's location. Each node starts a 3D scan using the available mechanical steering mechanism to achieve LOS with the neighbor node and perform a three-way handshake to establish the desired communication link between them. Considering the cone shape of the beam, a node can scan a spherical volume of radius $r$ (transmission range) around itself. This spherical volume can be considered as a combination of several circles with the same center as the sphere. Node A, randomly selects a circle to start scanning with an angular speed chosen from a given range. At the same time, it starts sending 'Hello' messages and keeps listening for an 'H-Ack' as response. Node B, similarly, chooses a circular path randomly, to scan with an angular speed chosen from a predefined range. Node B starts listening for 'Hello' messages at the same time. After a given time interval $T_{\text {newpath }}$, if Node A does not receive a response, it changes its transceiver's circle of rotation. After another time interval $T_{\alpha}$, it changes its angular speed too. Node B independently does the same. This continues until the nodes' transceivers are within each other's LOS. When the transceiver beams of the nodes start crossing over each other, if the 'Hello' is received by Node B. And, Node B responds with the 'H-Ack' and waits for an 'Ack'. Node A receives this ' $\mathrm{H}$-Ack', sends the 'Ack' and stops the scanning process. Node B receives the 'Ack' and also stops the transceiver rotation and brings an end to the three-way handshake. This completes the neighbor discovery process and a highly directional communication link is established.

\section{IN-BAND LINE-OF-SIGHT DISCOVERY}

In this section, our proposed LOS discovery algorithm will be discussed in detail. The important parts of the algorithm are, to select the circular paths, when to move to a new path, the angular speeds of the transceivers' rotations and when to reset the angular speeds.

\subsection{Randomized selection of Great Circles}

Since the nodes are not aware of each other's location and have no omni-directional communication channel, a complete scan of the surrounding space is necessary for neighbor discovery. Considering a node with a transceiver of communication range $R_{\max }$, the volume covered by this transceiver's beam can be considered to be a sphere of radius $R_{\max }$ with the node's position as the center. This sphere can be considered as a combination of a number of Great Circles [4]. So, the scanning of the whole spherical volume can be approximated by scanning through multiple of these Great Circles (e.g., similar to a ball covered with thread).

The following set of equations provide a circle in the $x-y$ plane with center at $(0,0,0)$, radius of $R_{\max }$, zenith angle $\theta$ and azimuth angle $\phi$. 


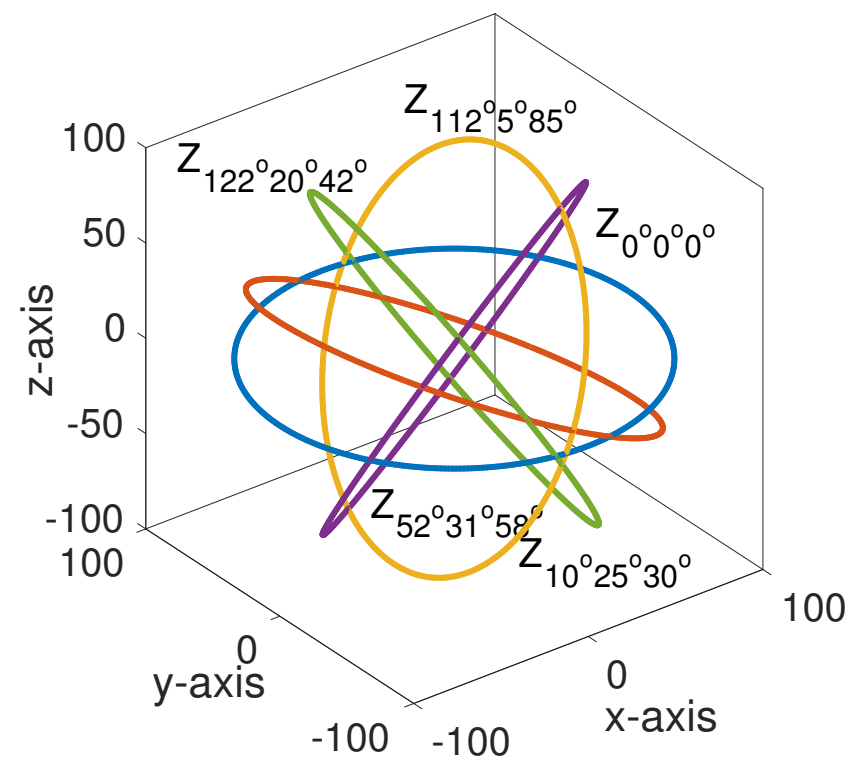

Figure 1: Great Circles generated using rotation matrices

$$
\left\{\begin{array}{l}
\theta=90^{\circ}, 0 \leq \phi<360^{\circ} \\
x=R_{\text {max }} \sin \theta \cos \phi \\
y=R_{\text {max }} \sin \theta \sin \phi \\
z=R_{\text {max }} \cos \theta
\end{array}\right.
$$

Now, the other Great Circles on the same sphere with center at $(0,0,0)$ and radius $R_{\max }$ can be generated by using the following rotation matrices:

$$
\left\{\begin{array}{l}
R_{x}=\left[\begin{array}{ccc}
1 & 0 & 0 \\
0 & \cos \theta_{x} & -\sin \theta_{x} \\
0 & \sin \theta_{x} & \cos \theta_{x}
\end{array}\right] \\
R_{y}=\left[\begin{array}{ccc}
\cos \theta_{y} & 0 & \sin \theta_{y} \\
0 & 1 & 0 \\
-\sin \theta_{y} & 0 & \cos \theta_{y}
\end{array}\right] \\
R_{z}=\left[\begin{array}{ccc}
\cos \theta_{z} & -\sin \theta_{z} & 0 \\
\sin \theta_{z} & \cos \theta_{z} & 0 \\
0 & 0 & 1
\end{array}\right]
\end{array}\right.
$$

Here, $R_{x}, R_{y}$ and $R_{z}$ represents rotations around the $x, y$ and $z$ axes, by angles of $\theta_{x}, \theta_{y}$ and $\theta_{z}$ respectively. The value of each of these angles is picked randomly from the range $\left[0,180^{\circ}\right)$. So, using (1) and (2) in the following equation, we get the equations of the other Great Circles:

$$
Z_{\theta_{x} \theta_{y} \theta_{z}}=\left[\begin{array}{l}
x^{\prime} \\
y^{\prime} \\
z^{\prime}
\end{array}\right]=R_{z} R_{y} R_{x}\left[\begin{array}{l}
x \\
y \\
z
\end{array}\right]
$$

Figure 1 shows an example of such Great Circles. For instance,

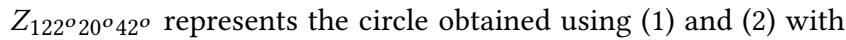

$\theta_{x}=122^{\circ}, \theta_{y}=20^{\circ}$ and $\theta_{z}=42^{\circ}$.

\subsection{Choosing the angular speed}

Once a random Great Circle is chosen for a transceiver to scan through, the next thing to decide is the angular speed of this scan. The angular speed should be chosen in such a way that the nodes get enough time to complete the three-way handshake to discover each other. The time required to complete the three-way handshake $(\tau)$ can be calculated as:

$$
\tau=t_{t r n}+3 \times t_{p r p}+2 \times t_{p r c}
$$

Here, $t_{t r n}$ is the transmission delay that can be calculated by dividing the total size of Hello, H-Ack and Ack packets by the data rate. The propagation delay $\left(t_{p r p}\right)$ is the time required (in the order of nano seconds) for the signal to travel to the neighbor node. And $t_{p r c}$ is the processing delay that depends on the speed and load of a node's processor.

Now, the time required for a node's transceiver beam to cross over the neighbor node varies with the position of the neighbor. Figure 2 shows the side view and cross-section of a transceiver beam crossing a neighbor. Let us assume that the line $a c a^{\prime}$ represents a portion of the path followed by the transceiver beam. If the neighbor is situated somewhere on $a c a^{\prime}$, given an angular speed of $\omega$ and divergence angle of $\beta$, the time required for the beam to cross-over the neighbor would be $\frac{2 \beta}{\omega}$. Similarly, if the position of the neighbor is somewhere on $b b^{\prime}$ or $d d^{\prime}$, the time required by the transceiver beam to cross-over it would be $\frac{\lambda}{\omega}$ or $\frac{\lambda^{\prime}}{\omega}$ respectively. Here, $\lambda^{\prime}<\lambda<$ $2 \beta$ which implies $\frac{\lambda^{\prime}}{\omega}<\frac{\lambda}{\omega}<\frac{2 \beta}{\omega}$. So, the node will face its neighbor the maximum duration of time when the neighbor's position is on the path followed by its transceiver beam. So, for successful neighbor discovery, this time duration must be greater than the time required for completing the three-way handshake. Thus, the relation between the angular speed $\omega$ and $\tau$ should be:

$$
\frac{2 \beta}{\omega} \geq \tau
$$

Hence, the angular speed must be chosen from the following range:

$$
0<\omega \leq \frac{2 \beta}{\tau}
$$

We will denote $\frac{2 \beta}{\tau}$ as $\hat{\omega}$ in the rest of the paper.

\subsection{Moving to a new path}

We have discussed how (1), (2) and (3) can be used to select a circular path for a node to scan through with its transceiver beam. Now, another important decision to make is, after what time period should a node move its transceiver beam to a new circular path, in other words, how long should a node scan through the current path. Let us denote the time period after which a node moves its transceiver to scan through a new circular path as $T_{\text {newpath }}$ and the average neighbor discovery time achieved using this $T_{\text {newpath }}$ is $t^{a v g}\left(T_{\text {newpath }}\right)$. So, the optimal value of $T_{\text {newpath }}$ would be:

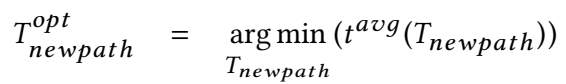




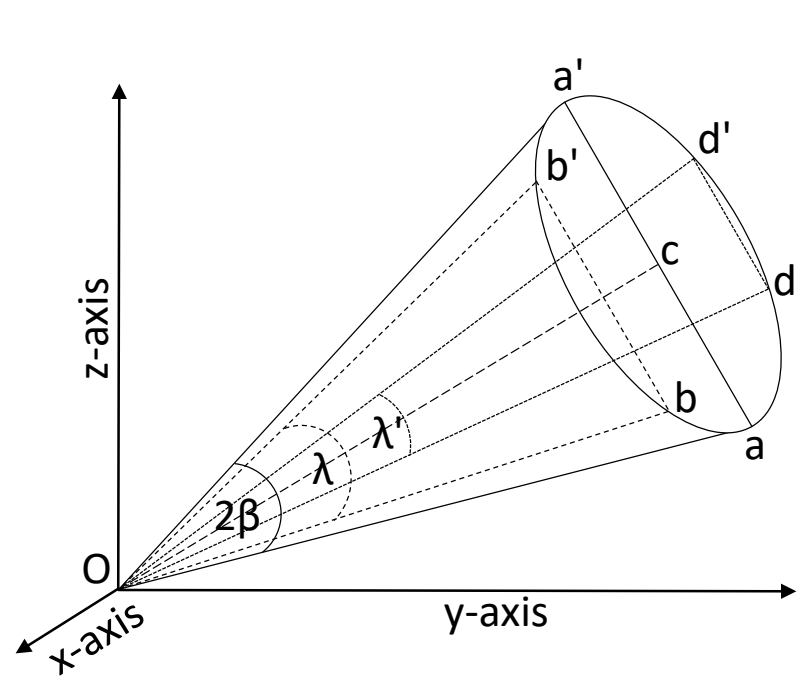

(a) Side view

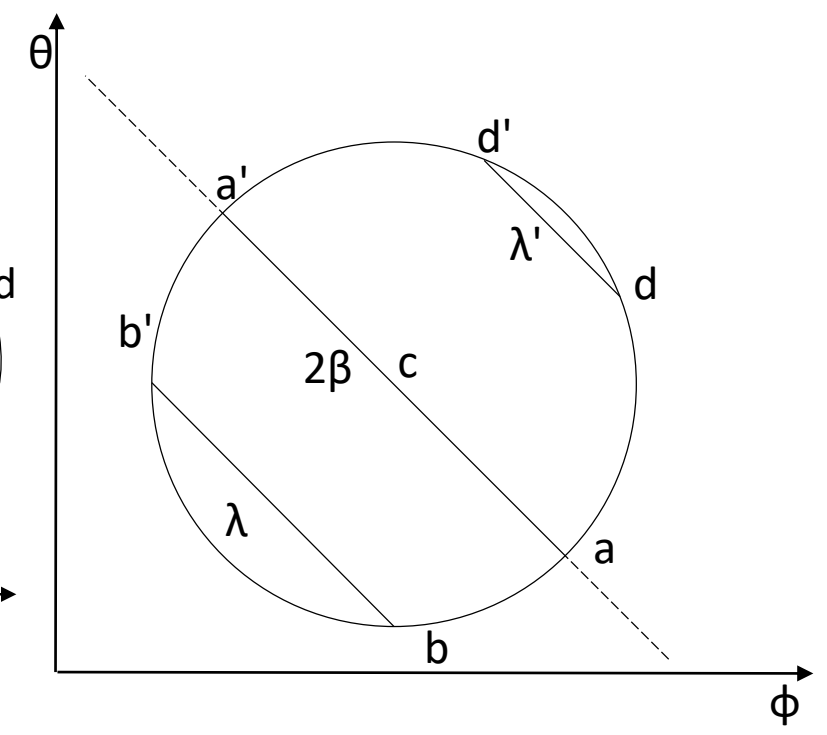

(b) Cross section

Figure 2: Scanning path of transceiver beam

So, the value of $T_{\text {newpath }}$ that provides the smallest average neighbor discovery time is chosen as the time period after which the transceiver beam is moved to a new path.

\subsection{Resetting the angular speed}

Another decision to make for a node is when to change the value of the angular speed $\omega$. The initially chosen value of $\omega$ may result in very large discovery times. For example, if both nodes choose the same angular speed and are facing their transceivers at the same direction at some point of time, the nodes will always be out of phase and will never discover each other. So, changing the angular speed after a given time period is very important. Let us assume that the probability of discovery within time $t$ is given by $p_{t}$ and the confidence level is given by $\alpha \in(0,1)$. Now, we can obtain the angular speed reset time $T_{\alpha}$ for a given confidence level $\alpha$ as follows:

$$
\begin{array}{rlr}
T_{\alpha}^{o p t}= & \underset{T_{\alpha}}{\arg \min }\left(t\left(T_{\alpha}\right)\right) \\
& \text { s.t. } \quad p_{t} \geq \alpha
\end{array}
$$

\subsection{Optimum range of angular speed}

As discussed earlier, the angular speed, $\omega$, chosen by a node must be within the range $\left(0, \frac{2 \beta}{\tau}\right)$ or $(0, \hat{\omega})$. This range may not give the optimal discovery time, so, a range $\left[\omega_{\min }, \omega_{\max }\right]$ is chosen for selecting the values of $\omega$. The optimum values of $\omega_{\min }$ and $\omega_{\max }$ are:

$$
\begin{array}{rll}
\omega_{\text {min }}^{\text {opt }}= & \underset{\omega_{\text {min }}}{\arg \min }\left(t^{a v g}\left(\omega_{\text {min }}, \omega_{\text {max }}\right)\right) \\
\omega_{\text {max }}^{\text {opt }}= & \underset{\omega_{\text {max }}}{\arg \min }\left(t^{a v g}\left(\omega_{\text {min }}, \omega_{\text {max }}\right)\right) \\
\text { s.t. } & \omega_{\text {min }} \in(0,2 \beta / \tau), \\
& \omega_{\text {max }} \in\left(\omega_{\text {min }}, 2 \beta / \tau\right]
\end{array}
$$

\section{SIMULATIONS AND RESULTS}

We performed simulations using MATLAB to gain insight into the effectiveness of the proposed neighbor discovery method. The nodes' positions were randomly chosen for each run of the simulation. The maximum communication range was fixed at $100 \mathrm{~m}$. We considered divergence angles of $5^{\circ}, 10^{\circ}, 15^{\circ}, 20^{\circ}$ and $25^{\circ}$. We assumed the nodes to be equipped with FSO transceivers (LEDs/VCSELs as transmitters and photodiodes as receivers). The received power and the communication range of an FSO transceiver is affected by Lambertian loss, atmospheric attenuation and geometric attenuation. Accordingly, we calculated the received power $P_{r c v}$ of a node's transceiver from the following relations [17]:

$$
\begin{gathered}
-\left(P_{t}+P_{r}\right)<10 \log _{10} e^{-\sigma R \cos \delta}+ \\
10 \log _{10}\left(\frac{\zeta}{\gamma+200 \beta R \cos \delta}\right)^{2} \\
P_{r c v}=P_{r} \times \cos \delta
\end{gathered}
$$

where, $R$ is the distance between the nodes in meters, $\delta$ is the radial distance of a node from its neighbor's beam's axis of propagation, $P_{t}$ is the transmitter's source power in $\mathrm{dBm}, P_{r}$ is the received power along beam normal in $\mathrm{dBm}, \gamma$ is the transmitter radius in $\mathrm{cm}, \zeta$ is the receiver radius in $\mathrm{cm}, \beta$ is the divergence angle of transmitter in mRad. 


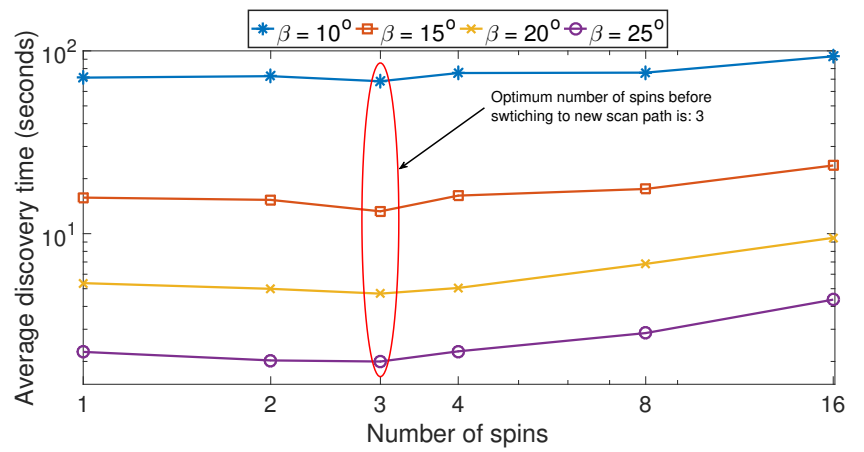

Figure 3: Average discovery time changing with number of spins

The messages used for the three-way handshake 'Hello', 'H-Ack' and 'Ack' were considered to be 38 bytes and data rate was $1 \mathrm{Mbps}$, which gives packet transmission time of $\approx 300 \mu \mathrm{s}$ [1]. Assuming a processing delay of $100 \mu \mathrm{s}$, from (4), we calculated $\tau=3 \times 300+2 \times$ $100=1100 \mu s$.

\subsection{Finding $T_{\text {newpath }}$}

First, we ran simulations to find out the optimal time after which a node should move to a new circular path. The angular speed, $\omega$, was selected randomly from the range $(0, \hat{\omega}]$ at the start and was kept the same. As mentioned earlier, $\hat{\omega}=\frac{2 \beta}{\tau}$. The results are shown in Figure 3 with both axes in log scale. Here, the term 'spin' represents one full scan of a circular path or a rotation of $360^{\circ}$. Thus, $T_{\text {newpath }}=$ Number of spins $\times \frac{360^{\circ}}{\omega}$. Each point in the figure is an average of 1000 simulation runs. It is clear from the figure that the average discovery time is minimum when the scanning path is changed after 3 scans or $T_{\text {newpath }}=3 \times \frac{360^{\circ}}{\omega}$. We can see that, as the number of spins increases from 1 to 3 , the average discovery time decreases. But, as number of spins increases to more than 3 , the average discovery time keeps increasing. And this trend can be observed for all values of the divergence angle, $\beta$. Moreover, we can observe that, the average discovery time increases as $\beta$ becomes smaller.

\subsection{Finding $\alpha$ and $T_{\alpha}$}

We performed more simulations to find out the angular speed reset time, $T_{\alpha}$, for a given confidence level, $\alpha$. The value of $T_{\text {newpath }}$ was calculated as $3 \times \frac{360^{\circ}}{\omega}$, where $\omega \in(0, \hat{\omega}]$. For each value of $\beta$, the simulation was run 1000 times. Figure 4 shows the empirical CDF (cumulative distribution function) of the discovery times. The inner box shows the CDF of discovery times with $x$-axis in logarithmic scale. The $y$-axis of this figure represents the confidence level $\alpha$. For $\beta=25^{\circ}, 99 \%$ of the time discovery is completed within $16.88 \mathrm{~s}$. For $\beta=20^{\circ}, 99 \%$ of time neighbor discovery is achieved within $31.43 \mathrm{~s}$. On the other hand, for smaller values of $\beta$ like $10^{\circ}$ and $15^{\circ}$ discovery takes longer time period. For example, with $\beta=10^{\circ}$, we can see that, $90 \%$ of the time neighbor is discovered within $\approx 154 \mathrm{~s}$. So, we set the angular speed reset time $T_{\alpha}$ as $154 \mathrm{~s}$ for confidence level of $90 \%$ or $\alpha=0.9$ for transceivers with $\beta=10^{\circ}$. The values of $\alpha$ and $T_{\alpha}$ for different $\beta$ are shown in Table 1.

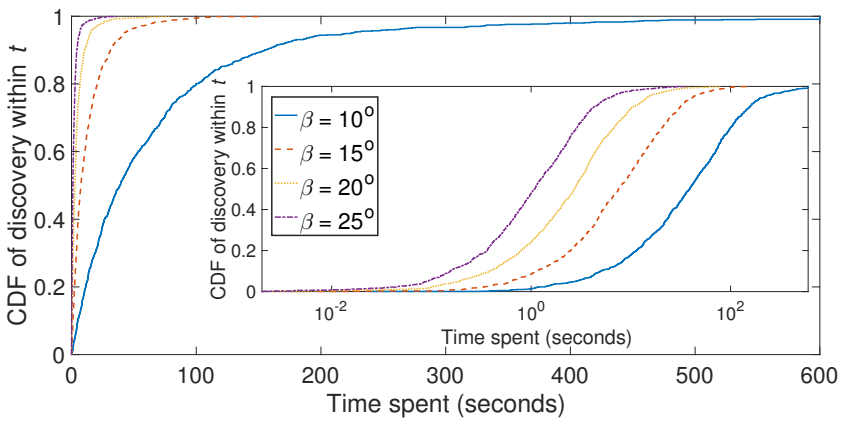

Figure 4: Empirical cumulative probability of discovery

Table 1: Angular speed reset time $T_{\alpha}$ in seconds

\begin{tabular}{cccccc}
\hline \multirow{2}{*}{$\alpha$} & \multicolumn{5}{c}{$T_{\alpha}$} \\
\cline { 2 - 6 } & $\beta=5^{\circ}$ & $\beta=10^{\circ}$ & $\beta=15^{\circ}$ & $\beta=20^{\circ}$ & $\beta=25^{\circ}$ \\
\hline 0.1 & 85.82 & 4.93 & 1.11 & 0.38 & 0.16 \\
0.2 & 184.87 & 11.40 & 2.48 & 0.79 & 0.36 \\
0.3 & 299.24 & 18.90 & 3.85 & 1.32 & 0.54 \\
0.4 & 429.83 & 26.38 & 5.27 & 1.920 & 0.76 \\
0.5 & 625.60 & 38.72 & 7.54 & 2.70 & 1.09 \\
0.6 & 827.07 & 53.34 & 10.57 & 3.59 & 1.52 \\
0.7 & 1097.30 & 74.27 & 14.57 & 4.67 & 2.12 \\
0.8 & 1557.20 & 99.78 & 20.72 & 6.827 & 2.89 \\
0.9 & 2663.40 & 154.84 & 31.23 & 10.38 & 4.24
\end{tabular}
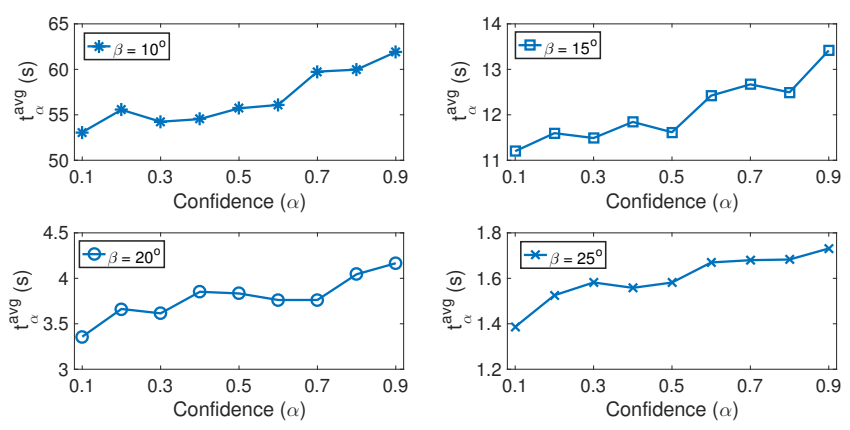

Figure 5: Average discovery times for different levels of confidence

We used the angular speed reset times $\left(T_{\alpha}\right)$ from Table 1 for different confidence levels $(\alpha)$ and performed further simulations. The results are displayed in Figure 5, where, we can observe that for transceivers of different divergence angles, the average discovery times are minimum when $\alpha=0.1$.

\subsection{Finding optimal $\omega_{\min }$ and $\omega_{\max }$}

In this section, our goal is to observe if changing the range $\left[\omega_{\min }, \omega_{\max }\right]$ from which the angular speed, $\omega$ is randomly chosen affects the performance of the algorithm. Here, we performed simulations for different combinations of $\omega_{\min } \in(0, \hat{\omega})$ and $\omega_{\max } \in\left(\omega_{\min }, \hat{\omega}\right]$. The divergence angle was $25^{\circ}$, angular speed was not reset and we used $T_{\text {newpath }}=3 \times \frac{360^{\circ}}{\omega}$. A selected portion of the result is 


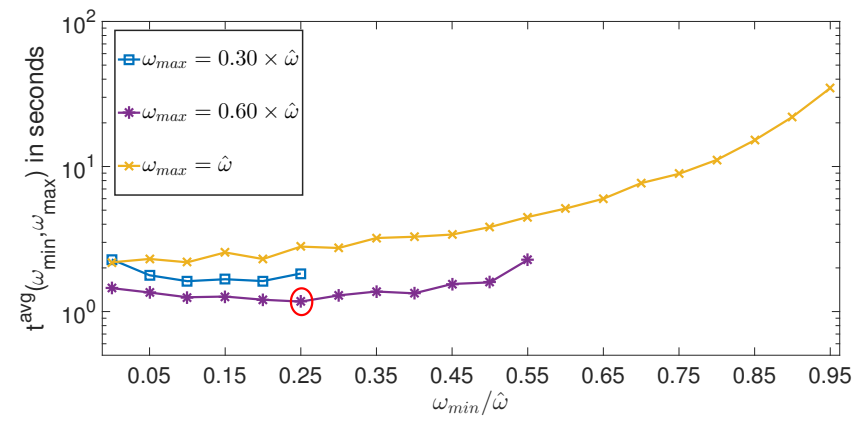

Figure 6: Average discovery time for different combinations of $\omega_{\min }$ and $\omega_{\max }$ with $\beta=25^{\circ}$

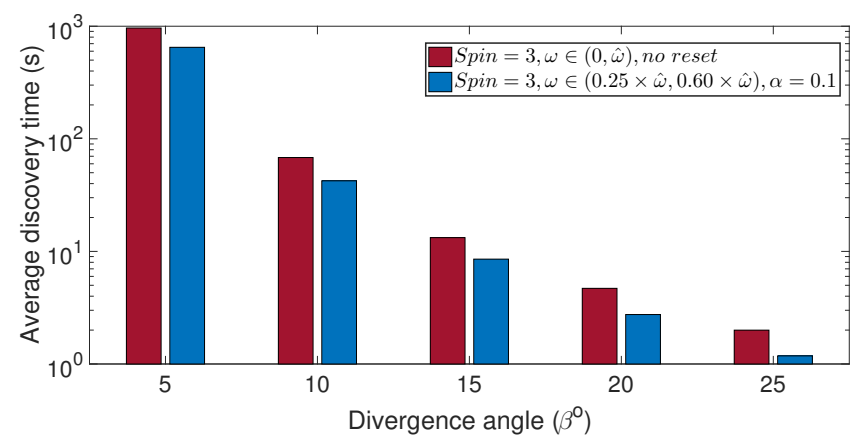

Figure 7: Significant improvement of average discovery time is achieved using $T_{\text {newpath }}, T_{\alpha}^{o p t}$ and $\omega \in\left[\omega_{\text {min }}^{o p t}, \omega_{\text {max }}^{o p t}\right]$,y-axis in logarithmic scale

displayed in Figure 6. The minimum average discovery time was observed for $\omega_{\min }=0.25 \times \hat{\omega}$ and $\omega_{\max }=0.60 \times \hat{\omega}$.

\subsection{Combined effect of $T_{\text {newpath }}, T_{\alpha}^{o p t}$ and $\left[\omega_{\text {min }}^{o p t}, \omega_{\text {max }}^{o p t}\right]$}

The results in the preceding sections provided the optimal value of $T_{\text {newpath }}=3 \times \frac{360^{\circ}}{\omega}, \alpha=0.1$ to select angular speed reset time and $\omega \in(0.25 \times \hat{\omega}, 0.60 \times \hat{\omega})$. In this section, we performed simulations using these values to observe how they affect the average discovery time. We compare the results to the case, where we choose $\omega \in(0, \hat{\omega})$ and without resetting the angular speed. Figure 7 demonstrates that significanlty smaller discover times can be achieved by using $\omega \in(0.25 \times \hat{\omega}, 0.60 \times \hat{\omega})$ and resetting the angular speed after every $T_{0.1}$ seconds. For example, for $\beta=15^{\circ}$, the average discovery time improves from $13.25 \mathrm{~s}$ to $8.53 \mathrm{~s}$, and for $\beta=25^{\circ}$, the average discovery time improves from $2 \mathrm{~s}$ to $1.2 \mathrm{~s}$. In all cases, the average discovery time reduces by more than $30 \%$.

\section{CONCLUSION}

In this paper, we proposed a novel LOS discovery method for drones situated in a 3D environment using only one directional transceiver. There is neither GPS nor any additional communication channel available. The drones can scan the surrounding environment using the directional transceiver in order to discover LOS with a neighbor drone. Through extensive simulations, we demonstrated that the proposed method can ensure successful neighbor discovery with very high probability. We observed that $\approx 1 s$ average discovery time is attainable for transceivers with $25^{\circ}$ divergence angle and rotating at a maximum speed of $0.6 \times \hat{\omega}^{o} / \mathrm{s}$. We showed that choosing a random angular speed from an optimum range and resetting it after optimal time intervals significantly reduces the discovery time. We plan improve the proposed algorithm to address the problem of LOS discovery among more than two nodes. Moreover, we plan to build a proof-of-concept prototype and conduct real test-bed experiments to gain more insight into the effectiveness of the proposed LOS discovery method.

\section{ACKNOWLEDGMENTS}

This work was supported in part by NSF award 1663764 and NIST award 70NANB17H188.

\section{REFERENCES}

[1] Suman Bhunia, Mahmudur Khan, Shamik Sengupta, and Murat Yuksel. 2016. LOS discovery for highly directional full duplex RF/FSO transceivers. In Military Communications Conference, MILCOM 2016-2016 IEEE. IEEE, 337-342.

[2] Lin Chen, Yong Li, and Athanasios V Vasilakos. 2016. Oblivious neighbor discovery for wireless devices with directional antennas. In INFOCOM 2016-The 35th Annual IEEE International Conference on Computer Communications, IEEE. IEEE, $1-9$.

[3] Romit Roy Choudhury, Xue Yang, Ram Ramanathan, and Nitin H Vaidya. 2002. Using directional antennas for medium access control in ad hoc networks. In Proceedings of the 8th annual international conference on Mobile computing and networking. ACM, 59-70.

[4] Great Circles. 2018. https://en.wikipedia.org/wiki/Great_circle.

[5] Facebook. 2015. Internet.org by Facebook. https://internet.org/.

[6] Google. 2017. Project Loon. https://x.company/loon/.

[7] Gentian Jakllari, Wenjie Luo, and Srikanth V Krishnamurthy. 2007. An integrated neighbor discovery and MAC protocol for ad hoc networks using directional antennas. IEEE Transactions on Wireless Communications 6, 3 (2007).

[8] Bruce E Johnson, Thomas A Lindsay, David L Brodeur, Randall E Morton, and Mark A Regnier. 1994. Wide-angle, high-speed, free-space optical communications system. US Patent 5,359,446.

[9] Mahmudur Khan, Suman Bhunia, Murat Yuksel, and Shamik Sengupta. 2016. LOS discovery in 3D for highly directional transceivers. In Military Communications Conference, MILCOM 2016-2016 IEEE. IEEE, 325-330.

[10] Mahmudur Khan and Murat Yuksel. 2015. Autonomous alignment of free-spaceoptical links between uavs. In Proceedings of the 2nd International Workshop on Hot Topics in Wireless. ACM, 36-40.

[11] Dongkyu Kim, Haesoon Lee, and Daesik Hong. 2015. A survey of in-band full-duplex transmission: From the perspective of PHY and MAC layers. IEEE Communications Surveys \& Tutorials 17, 4 (2015), 2017-2046.

[12] E Leitgeb, K Zettl, S Sheikh Muhammad, N Schmitt, and W Rehm. 2007. Investigation in free space optical communication links between unmanned aerial vehicles (UAVs). In Transparent Optical Networks, 2007. ICTON'07. 9th International Conference on, Vol. 3. IEEE, 152-155.

[13] Ram Ramanathan, Jason Redi, Cesar Santivanez, David Wiggins, and Stephen Polit. 2005. Ad hoc networking with directional antennas: a complete system solution. IEEE fournal on selected areas in communications 23, 3 (2005), 496-506.

[14] Sudarshan Vasudevan, Jim Kurose, and Don Towsley. 2005. On neighbor discovery in wireless networks with directional antennas. In INFOCOM 2005. 24th Annual foint Conference of the IEEE Computer and Communications Societies. Proceedings IEEE, Vol. 4. IEEE, 2502-2512.

[15] Yu Wang, Shiwen Mao, and Theodore S Rappaport. 2017. On directional neighbor discovery in mmwave networks. In Distributed Computing Systems (ICDCS), 2017 IEEE 37th International Conference on. IEEE, 1704-1713.

[16] Dyke Weatherington and U Deputy. 2005. Unmanned aircraft systems roadmap, 2005-2030. Deputy, UAV Planning Task Force, OUSD (AT\&L) (2005).

[17] Murat Yuksel, Jayasri Akella, Shivkumar Kalyanaraman, and Partha Dutta. 2009. Free-space-optical mobile ad hoc networks: Auto-configurable building blocks. Wireless Networks 15, 3 (2009), 295-312.

[18] Zhensheng Zhang and Bo Li. 2008. Neighbor discovery in mobile ad hoc selfconfiguring networks with directional antennas: algorithms and comparisons. IEEE Transactions on Wireless Communications 7, 5 (2008). 